\title{
Desempeño de la Defensoría Pública del Ecuador desde el Modelo Principal-Agente
}

\author{
Performance of the Public Defense of Ecuador from the \\ Principal-Agent Model
}

\author{
René Antonio Gálvez Delgado \\ Investigador Jurídico, SISEP \\ Artículo Original (Revisión) \\ RFJ, No. 2, 2017, pp. 353-376, ISSN 2588-0837
}

RESUMEN: en este artículo se efectúa un análisis del desempeño de la defensoría pública del Ecuador con el objetivo de identificar factores administrativos que afectan la eficiencia de esta institución para proponer soluciones que permitan una mejora en su rendimiento ya que, en los últimos años, se ha evidenciado la falta de productividad en este organismo. Esto resulta pernicioso si se considera que de su actuación depende el ejercicio de los Derechos de muchas personas en el país. En este documento, a través de la introducción del modelo principal-agente se explica la trascendencia de los costos de agencia en los resultados de la labor de la defensoría pública, como gestor, y los perjuicios que recibe la ciudadanía como principal. Además, se analizan algunos incentivos para combatir el problema de agencia en instituciones públicas y finalmente, se llega a la conclusión que el desempeño ineficiente de la defensoría pública, radica en un problema estructural de la administración ecuatoriana.

PALABRAS CLAVES: productividad, costos de agencia, defensa pública, litigio administrativo, incentivos.

ABSTRACT: in this article an analysis of the performance of the public defense of Ecuador is carried out with the objective of identifying administrative factors that affect the efficiency of this institution to propose solutions that allow an improvement in its performance since in recent years there has been a lack of productivity In this body, which is pernicious to consider that the exercise of the rights of many people in the country depends on their actions. In this project, through the introduction of the principal-agent model, the importance of the costs of agency in the results of the work of the public defense, as 
manager, and the damages that the citizenship receives as principal is explained. Some incentives to combat the problem of agency in public institutions are analyzed and finally the conclusion is reached that the inefficient performance of public defense lies in a structural problem of the Ecuadorian public administration.

KEY WORDS: productivity, costs of agency, public defense, administrative litigation, incentive.

\section{INTRODUCCIÓN:}

La concepción occidental del fin último del Estado ha variado desde sus inicios, actualmente es ampliamente aceptada la idea que el Estado debe ser un ente garante de Derechos hacia la ciudadanía.

El Ecuador no es la excepción, de hecho desde la promulgación de la Constitución de Montecristi la garantía de los Derechos ha sido un tema prioritario, al menos en materia legal, uno de los Derechos tutelados es la carta magna es el Derecho a la defensa legal de manera gratuita, para ello se instituyó un órgano que pese a tener algunos antecedentes similares en el país es fue novedoso en su momento; esta es la defensoría pública del Ecuador, al momento de su creación fue idealizada como una entidad que brinde un servicio de defensa gratuito y de calidad, sin embargo, su labor no es considerada óptima por parte de la ciudadanía y ha recibido reiteradamente varias críticas respecto a la calidad de su desempeño.

La motivación de este trabajo consiste precisamente en buscar posibles soluciones a esta problemática ocasionada ya que el esfuerzo que invierten los abogados en los casos presentados por los usuarios de la defensoría pública no es el mismo ni tiene la misma eficacia que el invertido por un abogado particular, en relación con su cliente, para analizar este problema es necesario entender por partes la relación que se establece entre los beneficiarios de los servicios de la defensoría pública del Ecuador y los servidores públicos que laboran en ella, para ello, se utilizará el modelo principal-agente el cual permite tener una visión más amplia del vínculo que existe entre ambas partes y los elementos causantes de la productividad deficiente de un empleado entendida como los costos de agencia. 
En este trabajo se pretende realizar un análisis de la labor realizada por la defensoría pública e identificar los problemas de agencia que se presentan en esta institución para poder identificar falencias que son comunes dentro de los organismos estatales precisamente por el diseño del sistema en el que tienen que desarrollan sus actividades, un sistema propicio para que existan actuaciones arbitrarias, que la mayor parte del tiempo pasan inadvertidas ante el Estado y sus organismos de control y monitoreo, que, dicho sea de paso, tampoco se encuentran libres de problemas de agencia. A través del modelo principal-agente se procederá a evaluar otros elementos como el diseño de la estructura institucional mediante un abordaje a los fundamentos legales de la defensoría pública del Ecuador y los resultados que la actuación de este organismo ha generado, para este punto se realizará una revisión de los informes de labores de la defensoría pública del Ecuador correspondientes a los años 2014 y 2016, de igual manera se ha considerado para este punto las opiniones de la ciudadanía respecto a la defensoría pública del Ecuador plasmadas en confiables medios de comunicación, y algunos datos estadísticos proporcionados por instituciones gubernamentales e internacionales.

La finalidad de este trabajo es proporcionar una visión más amplia acerca del Estado de los organismos de gobierno ecuatorianos en general y de la defensoría pública del Ecuador en particular para, a lo largo de este escrito encontrar posibles soluciones a los problemas de agencia y la baja productividad estatal que afecta directamente a la ciudadanía.

Este artículo se compone de tres secciones orientadas a tres objetivos específicos planteados al inicio de la investigación, la primera sección desarrolla el primero de ellos que consiste en realizar una descripción del problema de agencia y las implicaciones que tiene en el sector público, el segundo objetivo implica realizar un análisis estructural de la defensoría pública del Ecuador mediante una revisión de su normativa, para ello en la segunda sección se procede a llevar a cabo este cometido, finalmente se presenta una tercera sección en donde se evalúa el desempeño de la defensoría pública del Ecuador mediante su informe de labores del año 2016 en concordancia con el tercer objetivo planteado. 


\section{MODELO PRINCIPAL-AGENTE Y LOS COSTOS DE AGENCIA}

Para iniciar es necesario comprender que es el modelo principal agente. El mismo de una manera simplificada consiste en la ilustración de la relación que existe entre dos partes en el contexto una delegación de determinada tarea.

El modelo principal agente es el vínculo que existe entre dos sujetos, un primer sujeto denominado principal, y un segundo sujeto denominado agente o gestor, en donde los beneficios que obtenga el principal son directamente proporcionales a la calidad del desempeño del agente ${ }^{254}$, el principal procede a la contratación de un agente para la realización de una labor que este por falta de tiempo o conocimientos no puede llevarla a cabo, el modelo principal-agente intenta explicar la actuación de los agentes económicos a base de su racionalidad. ${ }^{255}$

Este modelo es utilizado principalmente en las ciencias económicas y pese a gozar de gran utilidad en el ámbito empresarial y financiero ha logrado difundirse hacia otras áreas ya que facilita interpretar de mejor manera los costos de transacción, definidos como costos necesarios para ordenar o crear y operar las instituciones y garantizar la obediencia de las reglas ${ }^{256}$, Dichos costos se generan frecuentemente en relaciones jerárquicas tales como empresas y hasta el propio gobierno. En esta ocasión el modelo del principal-agente nos servirá para ilustrar el problema de agencia y sus efectos en la eficiencia de la defensoría pública del Ecuador.

El problema de agencia. Estos se presentan cuando hay una actuación poco productiva por parte del agente y como resultado se afectan negativamente los resultados deseados por el principal, la baja productividad del agente en muchas ocasiones es producida por la discrepancia de intereses entre ambos sujetos, en otras palabras, esperan un resultado distintito respecto a la realización de un fin.

La Teoría de Agencia explica los costos de agencia a través de tres variables ${ }^{257}$, estas variables resumen las divergencias entre el principal y el agente. La primera de estas es la incentivación, que consiste en

254 Posner, E. (2000). Law and economics.

255 Ganga Contreras, F. y Félix Burotto, J, (2017). 87.

256 Germán G. y Rodríguez B. (2011). 123.

257 Jensen y Mecling (1976). 
las motivaciones divergentes entre las partes, esto genera conflictos de intereses entre las mismas. Una de las dificultades que se presenta en una relación de agencia radica en que se da por sentado que el principal y el agente tienen las mismas metas y un interés ${ }^{258}$ común, pero en realidad esto no siempre ocurre, al contrario, los fines perseguidos por ambas partes casi siempre son diferentes, mientras el principal tiene un objetivo definido, el gestor se plantea otro muy distinto influenciado por sus aspiraciones personales o quizá la misma meta, pero matizada con sus propias expectativas. En este punto aparece otro concepto que es importante abordar el cual es el riesgo moral, cuando el agente se desempeña en consideración a sus intereses personales y deja pasar por alto los del principal, de esta forma disminuyen los beneficios finales del titular.

La segunda variable es la asimetría de información que complica el desempeño productivo de la relación ya que el principal y el agente actúan en escenarios de conocimiento desiguales y con información insuficiente, este hecho se presta a incrementar el problema de agencia ya que normalmente el agente se encuentra en una ventaja comparativa respecto del principal lo que puede ocasionar comportamientos oportunistas por parte del gestor en un intento por cumplir con su objetivo personal, por ende la relación contractual se torna ineficiente, con menores beneficios de los que podría obtener el principal. ${ }^{259}$

La tercera variable es el factor de riesgo a asumir; ambas partes tienen una predisposición distinta con respecto al riesgo a emplear para alcanzar el objetivo, clásicamente se suele dividir a los individuos en amantes al riesgo, neutrales al riesgo e individuos adversos al riesgo, en esta categoría se encuentra la mayoría de la población, el riesgo a tomar es determinante para la consecución de un objetivo, en la mayoría de casos las personas buscan empleos donde puedan tener estabilidad, especialmente salarial ya que de manera inconsciente esperan invertir un mínimo de esfuerzo pero además esperan contar siempre con una renta segura, esta brecha que existe entre el objetivo de estabilidad financiera con poco riesgo del gestor y el desempeño esperado de este por parte del agente es donde se originan los problemas de agencia.

258 Fernández Olmos, M. y Marín Vinuesa, L. (2012). 5.

259 Ganga Contreras, F. y Quiroz Castillo, J (2015). 641. 
Estos tres factores constituyen serios problemas que dificultan el desempeño efectivo del agente en un trabajo y se relacionan con otras categorías determinantes en las relaciones de agencia.

Aparte de los problemas ya mencionados se encuentran otros factores causantes de problemas de agencia como el desconocimiento del sujeto a contratar, aquí aparece el problema de la elección equivocada que se presenta en un contexto donde el agente no posee las actitudes, aptitudes o medios de conocimiento requeridos para el cumplimiento de la terea encomendada, este un inconveniente grave ya que en suma a las discrepancias de intereses que se presentan entre el principal y el agente se obtendrá un resultado ineficaz de la tarea encomendada por razones evidentes.

Pese a que el agente se encuentre consciente del mal desempeño de su trabajo, este realizará los esfuerzos necesarios para lograr mantener su puesto, estos comportamientos oportunistas no hacen más que empeorar la situación, por las razones expuestas es preciso mejorar de alguna manera la relación del principal y el agente.

A todo lo anterior hay que sumar una nueva dificultad y es que no hay que entender al modelo principal-agente como una relación únicamente entre dos individuos, las relaciones de agencia se extender a varios niveles; esto es conocido como problema de multiagencia ${ }^{260}$ en la cual las acciones del principal y los agentes superiores repercuten en los sujetos de menor jerarquía, esto es muy común a nivel de gobierno precisamente por el número de personar requeridas para el funcionamiento de sus instituciones.

Según García (2016) ${ }^{261}$ existen dos maneras para optimizar la actuación del agente, la primera consiste en una alineación de intereses entre ambos sujetos mediante un modelo de contratos que permita dicha finalidad, para lograrlo se deberían tener en cuenta las variables de la teoría de agencia (incentivación, asimetría de información y riesgo a asumirse), para generar un enfoque contractual más equitativo y eficiente entre las partes involucradas.

La segunda manera consiste en implementar un control eficaz que permita monitorear la actuación del sujeto gestor, el problema

260 González-Chavarría, A. 199.

261 García A. (2016). 4. 
con esta segunda opción radica en lo costosa que puede resultar, sin mencionar el hecho que al implementarla se pueden presentar más problemas de agencia, incluso en este punto Rumeau (2012) propone la existencia de una paradoja a la luz del modelo principal-agente ¿Quién controla a los controladores?.262

Una vez comprendidos los conceptos de problema de agencia y costos de agencia, resulta pertinente abordar el tema de los incentivos que inducen al gestor a actuar de una determinada manera. En este punto se analizará brevemente la manera en que ciertos factores contextuales en los que se ve inmerso el agente que determinan su manera de proceder cuando se le encarga la realización de una tarea.

En primer lugar se tiene que saber que los incentivos son los impulsos que hacen responsables para que la persona gestora invierta esfuerzo en una determinada proporción; estos están definidos tanto por las aspiraciones personales del sujeto como por el diseño del sistema en el que labora y como este le ayuda a lanzar sus objetivos, de esta manera se puede afirmar que la actuación del agente se encuentra determinada por los incentivos que recibe y estos a su vez por lo que espera del trabajo a ejecutar. De manera general, el escenario planteado líneas anteriores donde el gestor vela por sus objetivos y no invierte un esfuerzo extra suele ocurrir ya que el agente tiene un incentivo constante que es su salario, el mismo es percibido cada cierto tiempo y genera un ambiente rutinario que hace que el gestor entre en una zona de "confort" donde realizará el mínimo necesario, ubicado en un punto donde no sea despedido por el principal, pero que tampoco tenga que aplicar un esfuerzo mayor a su trabajo, esto es entendido desde el análisis económico del Derecho como "utilización de la racionalidad ante incentivos". ${ }^{63}$

Esto por inofensivo que parezca, es en muchas ocasiones un problema estructural y puede llegar en un nivel macro a afectar la productividad de toda una institución, una posible solución radica en utilizar incentivos para optimizar el rendimiento del contratado.

Los incentivos pueden ser muy variados y por esta razón se tiene que considerar que no se habla únicamente de dádivas o premios, también se refieren a acciones en contra del gestor como sanciones $\mathrm{u}$ otro elemento punitivo utilizado en su contra. A continuación, se presentan al-

262 Rumeau, D. (2012). 131.

263 Mongua, J. F. y Manrique Rodríguez, J. A. (2015). 141. 
gunos incentivos que pueden mejorar el desempeño de un agente enfocados desde una perspectiva jurídica, los mismos pueden ser aplicados al mismo tiempo, pero siempre con un enfoque adecuado y ordenado.

a) Incentivos de contratación: La idea de estos incentivos es que el trabajo cumpla con las expectativas tanto del principal como del agente, los mismos nacen de una reestructuración del enfoque de contratación de un organismo, esta clase de estímulos radican en la optimización de las formas de contratación con el fin de lograr una alineación de intereses entre el principal, y el agente, por esta razón su objetivo se concentra en las variables de incentivación y aplicación de riesgo, en este caso es necesario restructurar las condiciones de remuneración ${ }^{264}$, y dichas condiciones tienen que ser pactadas desde un inicio ${ }^{265}$, las cláusulas de los contratos deben fomentar la homologación de los intereses de ambas partes y definir desde el inicio las expectativas del contratante y el empleado.

Como ejemplo de esto se puede proponer la obtención de un valor extra al salario prefijado en proporcionalidad con los resultados obtenidos, claro que siempre será necesaria una base concreta de remuneración por la aversión al riesgo de la mayoría de individuos. El objetivo es planificar incentivos que estén diseñados de tal manera que se permita indagar los intereses del futuro agente y en la medida de lo posible direccionarlos al cumplimiento de la meta del principal, para dar cumplimiento a este postulado el Estado, para aplicar estos incentivos bien puede servirse de sus organismos de control para no solo detectar y sancionar anomalías sino también de recompensar actuaciones productivas y eficientes.

El departamento de recursos humanos tiene que ser más riguroso en la selección del personal para evitar el problema de la elección equivocada, los esquemas de contratación tienen que ser mucho más personalizados y no tan generales y homogeneizados como muchas instituciones lo manejan actualmente, especialmente en el sector público, para así lograr identificar aptitudes de manera individual, cada institución estatal debe contar con una planificación adecuada para alcanzar este fin. El establecer contrales más rigurosos para el ingreso a cargos públicos a su vez funciona para incentivar a los aspirantes a mejorar sus capacidades.

264 Pino-Pinochet, P., Ponce-Donoso, M., Carmen. y Vallejos-Barra, O. (2015). 123.

265 Pérez Hasbun, D. y Velazco Gutiérrez, D. (2015). 4. 
b) Incentivos de control y sanción: respecto al control de desempeño hay que mencionar que se encuentra presente dentro del Estado, su órgano más representativo es la Contraloría, encargada de velar por la transparencia y buen desempeño de funcionarios públicos, y en caso de una actuación adversa este organismo se encarga de aplicar sanciones ${ }^{266}$ con el objetivo de mejorar el sistema administrativo. Sin embargo, la falta de productividad por problemas de agencia no se refiere específicamente a actos ilegales sino de una actuación poco eficiente dentro del marco de la ley, sin embargo, es posible inducir una alerta del funcionamiento ineficiente de una institución mediante mecanismos que permitan que los agentes sientan la posibilidad de ser castigados ${ }^{267}$, mecanismos tales como legales como el litigio contra el Estado.

Según García (2016) el litigio puede ser utilizado como un sistema de alarma ${ }^{268}$, esto significa que la ciudadanía al sentirse insatisfecha con un servicio puede aplicar litigios en contra del respectivo organismo estatal, esto a largo plazo tiene un efecto de alerta que actúa directamente como un incentivo para una mejora continua del sistema; sin embargo, es importante destacar que este mecanismo depende del diseño de las reglas del sistema, en un escenario de reglas deficientes ${ }^{269}$, el litigio como sistema de alerta administrativa también los será.

En un escenario deseable, al imponer un litigio contra el Estado tienen que existir pocos obstáculos para iniciar el proceso para que el litigio sea visto como un hecho inminente por parte del órgano público, además el mismo tiene que ser eficaz, y objetivo, además en caso de determinar la responsabilidad del procesado tiene que existir una sanción real y palpable que servirá como estímulo para una mejora constante, una finalidad de este incentivo es utilizar los costos de agencia para conseguir que los funcionarios reaccionen ante estos y mejoren su labor. ${ }^{270}$

Como es evidente para alcanzar una aplicación exitosa de ambos incentivos es necesario que se den mejoras a la estructura de las insti-

266 Cordero Quinzacara, E. (2012). 134.

267 Castillo, M. y Balbinotto, G. (2017). 20.

268 García, A. (2016). 223.

269 Guzmán, X. (2012). 197.

270 Rabossi, M. (2014). 6. 
tuciones estatales, entendidas no solamente como los órganos del gobierno, también como las reglas de juego con las que se desenvuelven los mismos, para reducir los costos de agencia se tiene que realizar mejoras con una perspectiva integral, esto es no fijarse únicamente en el individuo como tal, sino identificar las falencias a nivel general.

Recordemos que la discordancia de intereses entre el principal y el agente no es el único dilema que se presenta en esta relación, también se da la deficiencia en el flujo de información, para este caso se requieren mecanismos que permitan un conocimiento más completo de las acciones del agente y que el principal posea las herramientas suficientes para monitorear su desempeño y de esta manera saber qué es lo que precisamente tiene que exigir al gestor.

\section{ANÁLISIS SITUACIONAL Y NORMATIVO DE LOS ÓRGA- NISMOS PÚBLICOS EN EL ECUADOR}

En el Ecuador se comenta reiteradamente sobre la ineficacia de sus instituciones públicas, y no es solo cuestión de percepción, cifras a nivel internacional revelan esta realidad, este es un problema que puede ser analizado desde muchas perspectivas, pero en esta ocasión nos ocupa el hacer una revisión de las deficiencias de los organismos estatales a la luz del modelo principal-agente y sus elementos.

Los problemas de agencia a nivel estatal en el Ecuador analizados desde las variables de la teoría de agencia parecen verse fuertemente marcados por la discordancia de intereses, aparentemente una gran mayoría de funcionarios públicos se mueven meramente por intereses personales ya que no existen controles adecuados que permitan modular sus acciones a los objetivos estatales, ${ }^{271}$ esto es corroborado en parte, en la alta tasa de corrupción que posee el Ecuador y los ineficientes resultados del accionar gubernamental en política económica y otras materias. Es indiscutible que el Ecuador tiene problemas administrativos en sus instituciones y esto repercute en la eficacia de la acción estatal.

Con respecto a la relación entre la corrupción y los costos de agencia no nos referimos a que el acto delictivo como tal y su reiterativo cometimiento constituye una causa de costos de agencia, pero es una clara

271 Mikhail Soto, I. y Cortez, W. (2015). 163. 
evidencia de la siguiente afirmación: Los cargos públicos en el Ecuador son vistos como un medio de lucro antes que un método para garantizar los Derechos de las personas, fin último del Estado ecuatoriano.

Con respecto a la variable de asimetría de información hay que destacar la importancia de optimizar el flujo de información para propiciar el control de desempeño y la rendición de cuentas, ${ }^{272}$ esto significa, realizar un monitoreo de la actividad del agente; como ya se mencionó este método resulta costoso, pero en un ámbito tan poco manejable como la administración pública y al realizar un análisis de costo-beneficio se concluye que resulta necesario.

Los organismos de monitoreo como ya se mencionó existen en el Estado pero para disminuir los costos de agencia en instituciones públicas resulta necesario un replanteamiento de sus funciones con el fin de evitar comportamientos oportunistas fundamentados de manera inconsciente en la estructura del sistema, algo común en el Ecuador, a continuación se desarrollará el porqué de esta afirmación, a breves rasgos se analizarán los factores administrativos y situacionales del país que influyen en que el servidor público tenga intereses dispares respecto a los objetivos estatales.

Los funcionarios de igual manera se ven influenciados por el nivel de riesgo que tomen, en el sector público los salarios suelen ser fijos y poco cambiantes por lo cual las personas, que en su mayoría son adversas al riesgo, prefieren estos puestos de trabajo antes que un empleo en el sector privado donde están sujetos a normas más personalizadas a voluntad de los particulares.

De esta manera, al comprender que el problema de eficiencia institucional no es un caso aislado del sistema en general, si no que forma parte de este es preciso hacer hincapié en una mejora de ese gran sistema administrativo del Estado.

Como se puede apreciar el escenario en el sector público se presta para la existencia de costos de agencia, precisamente porque el diseño del sistema es propicio para que prevalezcan los intereses particulares del agente sobre los de la ciudadanía que en última instancia es el principal, las numerosas relaciones de delegación de tareas y la inca-

272 Chamizo García, H. (2011). 9. 
pacidad estatal al intentar que funcionen da paso a una evidente crisis de las estructuras de gobernanza en el Ecuador. ${ }^{273}$

Es importante cuestionarse cuál es la raíz de este problema institucional en el país.

En este ámbito se observa un problema de agencia a nivel democrático, la ciudadanía como titular en teoría delega funciones a un gobierno que tiene la obligación de garantizar un óptimo funcionamiento del Estado, sin embargo, esto no sucede así, en el Ecuador no existen reglas de juego claras respecto al funcionamiento de sus organismos de administración estatal, el desenvolvimiento de los mismos se ve marcado en ocasiones incluso por los vaivenes políticos del país, la burocracia de los mismos es delegada por el gobierno de turno y esta se preocupa en priorizar el cumplimiento de su proyecto político antes que mantener una línea de certeza independiente del gobierno,

Las leyes son muy variables y cambiantes respecto al partido que ejerce el poder, por lo tanto, la institucionalidad se ve seriamente afectada, este fenómeno por una parte es responsable de la poca seguridad jurídica con la que cuenta el país, la seguridad jurídica tiene que ser sinónimo de estabilidad y eso es precisamente lo que se encuentra en serias dificultades en el país para su realización, al hablar de estabilidad no nos referimos a que el modelo de desarrollo de la administración estatal tiene que ser una fiel copia de sus antecesores y predecesores, sino que tenga un mínimo de continuidad y se garantice un orden jurídico que resulte eficaz para el cumplimiento de los objetivos predefinidos a miras de lograr la satisfacción del ciudadano. ${ }^{274}$

Aparte de lo preciso que resulta que en el Ecuador existan instituciones estables y capaces de garantizar estabilidad en el manejo de la administración estatal, también se tiene que mencionar que la eficiencia jurídica es de vital importancia para que no aparezcan intereses particulares, en un contexto de inseguridad los servidores públicos sienten que se encuentran en un escenario donde quien es más sabio y conoce los "trucos" es el que sale adelante, este es un mal generalizado en Latinoamérica precisamente por la inestabilidad política y económica de la región.

273 González-Chavarría, A. (2014). 192.

274 Marinoni, L. (2012). 256. 
El Ecuador no es la excepción, estamos ante un Estado que no solamente se ve afectado su inestabilidad política si no que posee una Economía muy cambiante y sus acciones se ven marcadas por la perpetua inestabilidad financiera en la que se desarrolla el país, ${ }^{275}$ así se puede afirmar que los problemas de agencia de cualquier institución, insistimos, no es un mal particular sino que son el resultado de una serie de factores internos y externos de la realidad del país en general, en este punto se ha realizado un análisis dogmático y somero de los factores administrativos causantes de problemas de agencia, en el siguiente punto se tratará el caso ecuatoriano desde una perspectiva jurídica más central.

Como conclusión a este punto resulta oportuno el mencionar que el Ecuador requiere de una modernización de la organización gubernamental que se encuentre a la par de los cambios de la vida moderna, aislada de los intereses de cualquier proyecto político, para llevar a cabo acciones tales como el monitoreo eficiente, la alineación de intereses o cualquier otro intento por reducir costos de agencia es necesario que el Estado abandone modelos arcaicos de gobernanza y establezca una planificación objetiva a futuro. Este último es un elemento del cual ha carecido el país, una ruta clara y definida es importante para identificar a tiempo cualquier anomalía que pueda presentarse. se tiene que hacer una profunda revisión de los métodos que se emplean actualmente en la administración pública, cuestionarse sobre las influencias partidistas en la burocracia y dar paso a las nuevas tecnologías de la información y comunicación para mejorar de manera eficiente la colapsada administración de los organismos estatales ${ }^{277}$, sin embargo la modernización de la administración estatal no tiene que confundirse únicamente con la implementación de tecnología, también significa el realizar un análisis sobre la forma en la que trabajan las nuevas generaciones, evaluar la eficiencia en la actualidad del tradicional modelo vertical de gobernanza y adaptarlo a la nueva realidad de hoy en día, hay que recordar que la alineación de intereses va de la mano respecto a cómo el servidos público se siente en su trabajo, para ello hay que ampliar el campo mucho más allá, inclusive al ámbito de la seguridad social para de esta manera implementar modelos de desarrollo más flexibles ${ }^{278}$, para obtener un sistema que no descuide ninguno de sus sectores, los problemas de agencia tienen

275 Tsuyoshi, Y. (2013). 9.

276 Zamora Torres, A. y Navarro Chávez, J. (2014). 121.

277 Secretaría de la Función Pública, Estados Unidos Mexicanos. (2015). 241.

278 Beristain Gallegos, E. (2013). 4. 
que ser abordados desde una perspectiva en la cual se vea la administración pública como una máquina, que no funciona si hay un defecto en alguna de sus piezas.

Al continuar con los postulados ya mencionados en los puntos anteriores en esta ocasión resulta oportuno un análisis del problema desde sus elementos de fondo, en este caso, las reglas de juego con las que se desempeñan las instituciones ecuatorianas y los componentes de estas que pueden estar vinculados presuntamente a la producción de costos de agencia.

La institución por analizarse será la defensoría pública del Ecuador, dicho organismo ha sido seleccionado ya que por la labor que este realiza es uno de los más adecuados para ilustrar el problema de agencia, el funcionamiento y funciones de la defensoría pública del Ecuador se encuentran definidos en varios instrumentos legales que juntos constituyen la base legal que fundamenta a la defensoría pública del Ecuador, se procederá a un análisis de los puntos correspondientes a los objetivos y deberes de esta esta institución y se detallará las razones por las que su normativa puede ser causante de costos de agencia.

En primer lugar y por orden jerárquico encontramos a la Constitución de la República del Ecuador en la que se enuncia el "deber ser" de la defensoría pública.

La Constitución en su artículo 75 establece que "toda persona tiene Derecho al acceso gratuito a la justicia y a la tutela efectiva, imparcial y expedita de sus Derechos e intereses, y que en ningún caso quedará en indefensión" ${ }^{279}$, como es apreciable este objetivo constitucional es muy idealista y no define un camino claro a seguir por la institución, el mismo dispositivo legal en su 76, numeral 7, literal a, establece que "nadie podrá ser privado del Derecho a la defensa en ninguna etapa o grado del procedimiento"; y, en el literal g del mismo artículo garantiza a toda persona el Derecho a ser asistida en los procedimientos judiciales por una abogada o abogado de su elección, o por defensora o defensor público.

Dentro de la base legal de la defensoría pública encontramos algunos decretos que forman parte del proceso de conformación de este órgano estatal, es importante destacar que el mismo nació tal y como hoy día es como parte del proyecto político del gobierno de Rafael Correa; el ex-

279 Constitución de la República del Ecuador, 2008. 
presidente sentó las bases de lo que sería la defensoría pública mediante dos decretos en el año 2007 los mismo se detallan a continuación.

El Decreto Ejecutivo $\mathrm{N}^{\circ} 563$ del 17 de agosto de 2007 publicado en el Registro Oficial $\mathrm{N}^{\circ} 158$ de 29 de agosto del mismo año, creó la Unidad Transitoria de Gestión de Defensoría Pública Penal, adscrita a la Presidencia de la República, y consecutivamente se emite otro, el Decreto Ejecutivo NO 748, de 14 de noviembre de 2007, publicado en el Registro Oficial NO 220 de 27 de noviembre de 2007, adscribió la Unidad Transitoria de Gestión de Defensoría Pública Penal al Ministerio de Justicia, Derechos Humanos y Cultos, manteniéndose como una entidad desconcentrada, con independencia administrativa y financiera.

Aquí se encuentra uno de los grandes problemas que inclusive es reiterativo en varias instituciones estatales, el referente a la autonomía financiera, esto no ocurre en el Ecuador, los órganos gubernamentales dependen casi indispensablemente de los fondos que el gobierno asigne, este es un claro ejemplo del mal manejo que se ha dado a las instituciones definidas por la Constitución de esta manera incluso la función de participación ciudadana no cuenta con la mismo, esto principalmente por una falta de planificación para tomar decisiones de inversión en un tiempo establecido ${ }^{280}$; en referencia a la autonomía también se encuentra el artículo 191 de la Constitución, en el cual se establece que la Defensoría Pública es un órgano autónomo de la Función Judicial, cuyo fin es garantizar el pleno e igual acceso a la justicia de las personas, sin embargo, es cuestionable hasta qué punto este mandato ha sido cumplido si en la última década la autonomía de la misma función judicial se ha visto comprometida; la independencia tiene que ser no solamente monetaria, sino también política ya que los funcionarios públicos que allí se encuentren van a priorizar los intereses del Estado antes que los del ciudadano que recurra a este organismo en busca de defensa, este fue un caso frecuente en el gobierno de Rafael Correa cuando ciudadanos inconformes con la actividad estatal acudían a la defensoría pública y esta no cumplía su trabajo eficientemente, todo esto por la falta de autonomía política y financiera de la institución.

Una vez realizada la evaluación de los fundamentos legales de la defensoría pública del Ecuador se procederá al análisis de sus atribuciones consagradas en el artículo 286 del código orgánico de la función judicial.

280 Pérez Hasbun, D. y Velazco Gutiérrez, D. (2012). 8. 
La primera tarea de esta institución según este artículo es el garantizar "la prestación gratuita y oportuna de servicios de orientación, asistencia, asesoría y representación judicial, conforme lo previsto en este código, a las personas que no puedan contar con ellos en razón de su situación económica o social”, este es el gran objetivo de la institución pero en la realidad se observa que la selección de las personas que aquí ingresan muchas veces no realmente necesita el servicio tanto como otras, esto ya que se presentan casos de corrupción donde los funcionarios reciben coimas por permitir el ingreso a cualquier persona, aquí se ve un evidente caso de primacía del interés del agente por sobre el del principal, el monitoreo a la actuación de los gestores en esta entidad tiene que ser mucho más riguroso, pese a que resulta costoso, pero reiteramos, resulta necesario, cuando una estructura no es funcional la eficiencia radica en la reorganización. ${ }^{281}$

El artículo 286 también le exhorta a "garantizar el Derecho a una defensa de calidad, integral, ininterrumpida, técnica y competente." Para entender la falla en el cumplimiento de este mandato, primeramente, se tiene que mencionar que los funcionarios de la defensoría pública perciben un salario fijo, de esta manera su interés de estabilidad se ve cubierto y descuidan la calidad de la defensa que proporcionan, simplemente esperan resolver todos los casos que llegan hasta ellos con la mayor brevedad posible, inclusive se llega en ocasiones a asesorar arbitrariamente a fin de terminar rápidamente con el caso, acción que evidentemente no harían con un cliente particular, esto además de ir en contra de la ética profesional del abogado, ${ }^{282}$ por consiguiente también incumple con otro objetivo de la defensoría pública del Ecuador que es el "Garantizar que las personas que tengan a su cargo la defensa pública brinden orientación, asistencia, asesoría y representación judicial a las personas cuyos casos se les haya asignado, $\mathrm{y}$ velen por el respeto a los Derechos de las personas a las que patrocinen. En todo caso primará la orientación a los intereses de la persona defendida", con los factores mencionados anteriormente es certero que los intereses de la persona defendida y por ende de la ciudadanía que es el principal no primarán.

Por otro lado, en muchas ocasiones no se respeta la opinión del ciudadano y se le imponen condiciones que el funcionario decide motivado por el cumplimiento de intereses personales respecto a la persona

281 Díaz Perilla, V. (2011). 14.

282 Anríquez Novoa, A. (2016). 336. 
a defender, se ha presentado este caso especialmente en escenarios de demandas contra el Estado. Este escenario es muy común cuando no existe verdadera autonomía de la institución. Esto no es legal ya que va en contra de otra atribución conferida a la defensoría pública por el mismo artículo 268 del código orgánico de la función judicial el cual a la letra reza "garantizar la libertad de escoger la defensa de la persona interesada y solicitar, de ser necesario, una nueva designación a la Defensoría Pública", por esta razón se insiste tanto en la búsqueda de autonomía del ejecutivo, no solo por parte de la defensoría pública sino de toda la función judicial.

Al retomar el tema del riesgo asumido por el agente se encuentra una nueva problemática y un nuevo incumplimiento de otro objetivo de la defensoría pública, este es el "apoyar técnicamente a las personas que hacen sus prácticas preprofesionales en la Defensoría Pública”. Según testimonios de pasantes en instituciones públicas los mismos no reciben el apoyo necesario, esto, puede deberse a que los funcionarios ya en cargo sienten una amenaza inminente por parte de los nuevos postulantes justamente por sus intereses de mantener sus cargos y la aversión a tomar riesgos en el futuro, esto puede resultar perjudicial para el organismo ya que se priva a corto plazo de un personal que puede tener mejores aptitudes que los agentes en funciones.

\section{EVALUACION DE EFICIENCIA DE LA DEFENSORÍA PÚ- BLICA DEL ECUADOR}

Una vez que se ha realizado el análisis del modelo principal-agente y se ha analizado la base legal de la defensoría pública del Ecuador, en esta parte se abordará la parte más pragmática de este trabajo, nos referimos a los resultados que ha dejado hasta el momento este organismo, para este punto será de utilidad el informe de labores de la defensoría pública del año 2016 y algunas referencias a esta institución realizadas por medios de comunicación, especialmente en la sección de cartas al director para obtener opiniones de la ciudadanía en general que, como se ha podido observar es el principal en esta relación.

El informe de labores de esta institución realiza una comparación respecto a la labor realizada por la defensoría pública en el año 2015 y 2016, para ilustrar de mejor manera estos datos, previamente a su análisis, a continuación se presenta una tabla que esquematiza los mismos. 


\begin{tabular}{|l|c|c|}
\hline $\begin{array}{l}\text { Tabla 1. Desempeño de la defensoría pública del Ecuador en los años } \\
2015 \text { y } 2016\end{array}$ & Año 2016 \\
\hline Elemento analizado & Año 2015 & \\
\hline $\begin{array}{l}\text { Número de puntos } \\
\text { de atención a nivel } \\
\text { nacional. }\end{array}$ & 148 & 205 \\
\hline $\begin{array}{l}\text { Patrocinios penales } \\
\text { realizados }\end{array}$ & 128.049 & 158850 \\
\hline $\begin{array}{l}\text { Número de defenso- } \\
\text { res por cada 100.000 } \\
\text { habitantes. }\end{array}$ & 4 & 53.238 \\
\hline $\begin{array}{l}\text { Asesorías realizadas } \\
\text { Número de personas } \\
\text { de mediación de la } \\
\text { defensoría pública. }\end{array}$ & 40.635 & 4496 \\
\hline
\end{tabular}

Fuente: Elaboración propia.

Como se puede apreciar en la tabla anterior, respecto a los avances de la defensoría pública encontramos la ampliación de sus puntos de atención respecto al año 2015 de 148 a 205, dicha institución presenta este incremento como un logro, pero la cuestión radica en si realmente esto significa un cambio positivo y beneficioso o simplemente se trata de un mero aumento de la burocracia estatal.

Con un aumento en los puntos de atención no se garantiza que el servicio será más eficiente, el servicio de la defensoría pública no puede medirse por su cobertura a nivel nacional, si no por el cumplimiento eficiente de sus deberes y la satisfacción que genera en diversos sectores de la ciudadanía. Para poner un ejemplo de aquello, mediante una publicación en la sección cartas al director en diario El Universo, Ariruma Kowii Maldonado, integrante de una comunidad indígena ecuatoriana mencionó que esta institución no cumple con el precepto de pluralismo jurídico de la Constitución y que en varias ocasiones parece ser que sus acciones son motivadas por razones políticas, como es 
el caso de comunidades indígenas que piden defensa contra el Estado y estas casi siempre son ineficientes; esto claramente reduce la confiabilidad de las personas en la institución, situación preocupante ya que la legitimidad constituye una de las bases del Estado de Derecho. ${ }^{283}$

Otro punto en el cual la defensoría pública alega mejoría es en la creación de oficinas itinerantes, iniciativa que puede resultar beneficiosa para garantizar el acceso de las personas menos favorecidas a este servicio para cumplir con el principio constitucional de igual$\mathrm{dad}^{284}$, pero es importante que previamente se realice una correcta planificación para reducir considerablemente los costos de transacción requeridos ${ }^{285}$, y por qué no, los futuros problemas de agencia que pueden presentarse, y evitar que dicha planificación solo signifique un aumento en la burocracia.

Otro punto importante a abordar es el tema de la calidad del servicio prestado, según el informe de la defensoría pública correspondiente al año 2016, en dicho año el organismo procesó un total de 331033 casos; sin embargo, como se ha logrado observar en páginas anteriores, la mejoría parce estar entendida como un aumento numérico de la atención y no en la calidad.

El mencionado informe habla sobre un mejoramiento en materia de contratación inclusiva y en asignación presupuestaria por parte del gobierno central pero estos logros consisten una mera mejora de la institución que para tener efectos reales debe ser orientada en beneficio de la ciudadanía , en efecto, si la institución mejora el servicio también lo hará, pero el informe demuestra que las condiciones del organismo son aún complejas, por ejemplo explica que el número de defensores, al cierre del año 2016 la cantidad de defensores públicos era de apenas cinco por cada cien mil habitantes, esto representa un serio problema para dar cumplimiento al principio de celeridad y eficiencia que plantea este organismo. Esto puede parecer una contradicción al expresado en líneas anteriores cuando se afirmó que un aumento en los empleados significa más burocracia, pero justamente por esto razón se insiste en realizar una asignación objetiva y justificada de tareas.

283 García Sánchez, E. (2014). 339.

284 Restrepo Echavarría, R. (2015). 148.

285 Gorbaneff, Y., Cortes, A., Torres, S. y Yepes, F.J. (2013). 335. 
La importancia de no tener una burocracia descontrolada radica precisamente en evitar problemas de agencia, en este punto resulta pertinente acotar que los problemas de agencia no son provocados por el número de personas que integran una institución, sino más bien por el manejo que se da del personal.

Si el recurso humano se encuentra mal administrado se da paso al afloramiento de intereses individuales a causa del sentimiento generalizado de ineficacia del sistema ${ }^{286}$. Tal y como funciona hoy en día la defensoría pública en el Ecuador resultaría pertinente considerar un nuevo diseño de la institución desde sus bases legales ${ }^{287}$.

En conclusión, al explicar basados en su informe de labores que este organismo requiere de una reestructuración nos referimos a revisar nuevamente las funcionalidades y competencias que tiene consignadas, analizar si su diseño realmente permite la cristalización de sus objetivos y de manera muy especial evaluar sus estándares de contratación y los procedimientos con los que se desenvuelve para reducir los costos de agencia y mejorar el alcance de los Derechos fundamentales de los individuos en relación con la defensa pública ${ }^{288}$.

\section{CONCLUSIONES}

El desempeño de una institución se ve marcada fuertemente por la calidad del trabajo realizado por cada uno de sus elementos individuales, el modelo principal-agente es un método sencillo para la ilustración y esquematización del vínculo que se da entre los gestores y el titular a fin de identificar los factores que propician una baja productividad por parte de los primeros tales como los intereses dispares, la asimetría de información y el nivel de riesgo asumido por el agente, gracias a este modelo se ha podido constatar la existencia de costos de agencia en la defensoría pública del Ecuador que afectan directamente a los beneficios que debería obtener la ciudadanía como principal, los más importantes factores que propician costos de agencia se encuentran en la estructura misma de esta institución como es la normativa legal con la que fue constituida y con la que funciona actualmente, el diseño de su base legal se presta para la existencia de intereses in-

\footnotetext{
286 Carrillo Flórez, F. y Varela, S.D. (2013). 2.

287 Fix-Fierro, H. y Suárez Ávila, A. (2015). 163.

288 Eissa, S. (2013). 54.
} 
dividuales dispares al objetivo de la defensoría pública y no para la alineación de los mismos a los del principal, además no cubre muchos escenarios y fomenta una asimetría de información constante, la normativa interna de este organismo de igual manera no toma en cuenta la predisposición al riesgo de sus funcionarios y provoca que estos apliquen el mínimo esfuerzo necesario, en sus labores. De esta manera para obtener un mejor desempeño de la defensoría pública es necesario aplicar varias mejoras de fondo en la institución, estas mejoras tienen que ir orientadas a crear incentivos que permitan la alineación de intereses y reduzcan la asimetría de información, la defensoría pública del Ecuador requiere de un mejoramiento real y concreto, esto significa de su eficiencia y eficacia, no únicamente un cambio entendido como un crecimiento de su cobertura nacional o la ampliación de su burocracia, se tiene que optimizar la calidad del trabajo de este órgano para así cumplir con el precepto constitucional con el que fue constituido que es el garantizar los Derechos de las personas.

\section{REFERENCIAS BIBLIOGRÁFICAS}

Anríquez Novoa, A. (2016). Ética profesional del abogado: Normativa vigente en Chile. Ius et Praxis, Vol. 2. 331-372.

Beristain Gallegos, E. (2013). Flexiseguridad. Revista Latinoamericana de Derecho Social, Vol. 17. 3-29.

Carrillo Flórez, F. y Varela S.D. (2013). Hacia un Sistema Integrado de Defensa Jurídica Estatal: Situación y perspectivas de una política pública en Colombia. Revista del CLAD Reforma y Democracia, Vol. 56, 123-176.

Castillo, M., y Balbinotto, G. (2017). A model of desertion. from a principal-agent theory perspective. Cuadernos de Economía, Vol. 70. 19-47.

Cordero Quinzacara, E. (2012). El Derecho administrativo sancionador y su relación con el Derecho Penal. Revista de Derecho, Vol. 2. 131-157.

Chamizo García, H. (2011). Las relaciones de agencia en el primer nivel de atención de la salud: desafíos para la equidad en Costa Rica. Horizonte Sanitario, Vol. 10. 7-21. 
Díaz Perilla, V. (2011). Calidad de la ley, técnica legislativa y eficiencia administrativa. Prolegómenos. Derechos y Valores, Vol. 14. 147-163.

Eissa, S. (2013). Redefiniendo la defensa: Posicionamiento estratégico defensivo regional. Revista SAAP, Vol. 7. 41-64.

Fernández Olmos, M. y Marín Vinuesa, L. (2012). Estudio del agente-principal en la agricultura. El trimestre económico, 304, Vol. 941-964.

Fix-Fierro, H. y Suárez Ávila, A. (2015). Hacia una defensa pública de calidad. el nuevo diseño institucional de las defensorías públicas en las entidades federativas de la república mexicana. Cuestiones Constitucionales, Vol. 32. 157-200.

Ganga Contreras, F., Quiroz Castillo, J. y Silvia A. (2015). ¿Qué hay de nuevo en la teoría de agencia (TA)?. Prisma Social. 685-707

Ganga Contreras, F., y Félix Burotto, J. (2017). Asimetrías de información entre agente y principal de las universidades chilenas. Estudios Gerenciales, Vol. 28. 83-104.

García Sánchez, E. (2014). Por una democracia eficaz. Andamios. Revista de Investigación Social, Vol. 11. 337-339.

García. A, (2016). El problema de agencia, ¿Un problema que nos afecta a todos? Ingeniería Jurídica y económica.

García. A. (2016). Análisis económico de las reglas del litigio contra el Estado en Derecho y Economía. Una revisión de la Literatura Latinoamericana. Quito: Centro de publicaciones PUCE.

González-Chavarría, A. (2014). Análisis institucional multiagente: El problema de estructuración y agencia en la explicación de la emergencia de estructuras de gobernanza. Revista de Estudios Sociales, Vol. 49. 190-204.

Gorbaneff, Y., Cortes, A., Torres, S. y Yepes, F. (2013). Teoría de costos de transacción, formas de gobernación y los incentivos en Colombia: un estudio de caso. Estudios Gerenciales, Vol. 29. 332-338.

Marinoni, L. (2012). El precedente en la dimensión de la seguridad jurídica. Ius et Praxis, Vol. 18. 249-266. 
Mikhail Soto, I. y Cortez, W. (2015). La corrupción en la burocracia estatal mexicana. Revista de Economía Institucional, Vol. 17. 161-182.

Mongua, J. F. y Manrique Rodríguez, J. A. (2015). LA Constitución y el problema de la agencia en las sociedades de capital concentrado desde el análisis económico del Derecho. Prolegómenos. Derechos y Valores, Vol. 18. 135- 152.

Pérez Hasbun, D. y Velazco Gutiérrez, D. (2012). Sostenibilidad y autonomía financiera en empresas de producción social. Multiciencias, Vol. 12. 121-125.

Pino-Pinochet, P., Ponce-Donoso, M., Avilés-Palacios, C. y Vallejos-Barra, O. (2015). Mejoramiento de la productividad en una industria maderera usando incentivo remunerativo. Maderas. Ciencia y Tecnología, Vol. 17. 117-128.

Rabossi, M. (2014). Los costos de agencia en instituciones universitarias: el establecimiento de un marco analítico para su evaluación. Education Policy Analysis Archives/Archivos Analíticos de Políticas Educativas, Vol. 22. 1-18.

Restrepo Echavarría, R. (2015). El surgimiento de la democracia constitucional de Derechos y justicia en Ecuador. Revista del CLAD Reforma y Democracia, Vol. 61. 133-176.

Rodríguez B. y Germán G. (2011). La firma y los costos de transacción. Actualidad Contable Faces, Vol. 22. 120-133.

Rumeau, D. (2012). El control civil de las políticas públicas de defensa: un modelo de análisis basado en la teoría del principal - agente. Revista Uruguaya de Ciencia Política, Vol. 21. 129-151.

Secretaría de la Función Pública, Estados Unidos Mexicanos. (2015). La Modernización de la Organización Gubernamental. Revista del CLAD Reforma y Democracia, Vol. 63. 230- 272.

Tsuyoshi, Y. (2013). Inestabilidad financiera en América Latina desde la perspectiva Kaleckiana y Minskyana. Problemas del Desarrollo. Revista Latinoamericana de Economía, Vol. 44. 9-27. 
Villaseñor Goyzueta, C. (2011). Proporcionalidad y límites de los Derechos fundamentales. Cuestiones Constitucionales, Vol. 27. 399-408.

Ximena Guzmán P. (2012). Respeto y protección del Derecho a la defensa. Revista Boliviana de Derecho, Vol. 13, 189-202.

Zamora Torres, A. y Navarro Chávez, J. (2014). Eficiencia de la administración pública aduanera a través del modelo DEA. Confines de Relaciones Internacionales y Ciencia Política, Vol. 10. 117-135.

\section{Base Legal}

Constitución de la República del Ecuador, R.O 499, Quito, 2008.

Código Orgánico de la función judicial, R.O 544, Quito. 2009.

Decreto Ejecutivo N563, 17 de agosto de 2007 R.O 158, Quito, 2007.

Decreto Ejecutivo NO 748, 14 de noviembre de 2007, R.O 220, Quito, 2007.

Recibido: $13 / 08 / 2017$

Aceptado: 29/11/2017

René Antonio Gálvez Delgado: Investigador jurídico del Grupo SISEP.

Correo electrónico: renegalvez627@gmail.com 